\title{
Distribution and Accumulation of Selenium in Plants and Health Risk Assessment from a Selenium-Rich Area in China
}

\author{
Bing Zhao', Shoubiao Zhou ${ }^{1 *}$, Xiaoguo $\mathbf{W u}^{2}$, Kun Xing ${ }^{2}$, Yuanyuan Zhu ${ }^{3}$, \\ Ling $\mathrm{Hu}^{2}, \mathrm{Xu} \mathrm{\textrm {TaO } ^ { 2 }}$ \\ ${ }^{1}$ Key Laboratory of Biological Resources Conservation and Utilization, College of Life Sciences, \\ Anhui Normal University, Wuhu, Anhui, P. R. China \\ ${ }^{2}$ College of Environmental Science and Engineering, Anhui Normal University, Wuhu, Anhui, P. R. China \\ ${ }^{3}$ Jiangsu Bio-Engineering Research Centre of Se, Suzhou, Jiangsu, P. R. China
}

Received: 15 September 2017

Accepted: 20 November 2017

\begin{abstract}
In order to investigate the concentrations of selenium (Se) in plants of the Dashan Region, a typical Se-rich area of China, and to illuminate the daily dietary Se intake of residents in this region, 83 crop samples and 144 Chinese herb samples were collected. Total Se was analyzed in the edible portion of crops and the medical portion of Chinese herbs. The average concentrations of Se ranged from 100 to $3,100 \mu \mathrm{g} \mathrm{kg}^{-1}$ (dry weight/DW) in different crops and from 20 to $1,500 \mu \mathrm{g} \mathrm{kg}^{-1}$ in the Chinese herbs (DW). The crop that contained the highest concentrations of Se was radish, while maize contained the lowest levels. For the Chinese herbs, the highest concentrations of Se were found in Rumex japonicas, while Cape jasmine contained the lowest levels of Se. The average enrichment coefficients (ECs) were $6.1-300 \%$ in crops, and $1.6-117 \%$ in Chinese herbs. Among the crops, radish had the highest EC (300\%), while pumpkin had the lowest (6.1\%). Among the Chinese herbs, Sapium sebiferum had the highest EC (117\%), while Dicranopteris dichotoma had the lowest (1.6\%). Based on the composition of residents' daily diets, the estimated daily Se intake from crops was $282 \pm 20 \mu \mathrm{g}$ day $^{-1}$, and was about 5 times higher than the RDA value suggested by WHO $\left(55 \mu \mathrm{g} \mathrm{day}^{-1}\right)$. Although no selenosis incidents have occurred in the Dashan Region to date, the potential health risk caused by chronic exposure to high levels of Se cannot be ignored.
\end{abstract}

Keywords: Dashan Region, China, selenium, plant, daily dietary Se intake

\section{Introduction}

Selenium ( $\mathrm{Se})$ is a trace element that can function as an essential nutrient for humans and animals or as

*e-mail: zhoushoubiao@vip.163.com

an environmental toxicant [1-2]. Se deficiency can lead to human diseases such as Keshan disease (an endemic cardiomyopathy) and Kashin-Beck disease (a type of osteoarthritis) [3-5]. By way of contrast, toxicity of Se will occur at high concentrations due to the replacement of sulphur with Se in amino acids, resulting in incorrect folding of the protein and consequently nonfunctional 
proteins and enzymes [6-8]. Generally, Se is important to humans and animals as an antioxidant. Many studies have shown that proper intake of Se could play a vital role in anti-aging, repairing cells, improving human immunity, and preventing cancer [3-4, 9-10].

Diet is the major source of Se intake for the general population. Se enters the food chain through plants, which take it up from the soil [11]. In Finland, for example, approximately $30 \%$ of the average daily Se intake came from plants directly, and the other $70 \%$ intake came from plants indirectly, by the consumption of animal origin products through the plant-animal-human food chain [12]. Se contents in plants are highly dependent on the levels of Se in soil and the plant's ability to take up and accumulate Se. Some native plants had the ability to accumulate $\mathrm{Se}$ when grown in seleniferous soils [13]. These plants are called Se accumulators and include some species of Astragalus, Stanleya, Morinda, and Neptunia, which can accumulate hundreds to several thousand mg of Se $\mathrm{kg}^{-1}$ (dry weight/DW) in their tissues. In contrast, most plants contain less than $25 \mathrm{mg}$ of $\mathrm{Se} \mathrm{kg}^{-1}$ (DW) and do not accumulate $\mathrm{Se}$ even they grow in seleniferous soils. These plants are called Se nonaccumulators [13].

The Dashan Region is located in southern Anhui Province, China. It is known as an Se-rich area, together with the other two Se-rich areas: Enshi of Hubei Province and Ziyang of Shanxi Province [14-15]. Previous investigations have indicated that the average concentration of Se in soil of the Dashan Region was about 5 times higher than that of the mean value of China [15]. Although no selenosis incidents have occurred in the Dashan Region, the potential health risk caused by chronic exposure of Se through the food chain cannot be ignored.

To date, there has no research focus on the distribution and accumulation ability of Se in the plants of the Dashan Region. Furthermore, the potential health risks caused by chronic exposure of Se through the consumption of plants are still unknown. The specific objectives of this study are: 1) to determine concentrations of Se in the plant samples of the Dashan Region, 2) to calculate the enrichment coefficients (EC) and identify the accumulation ability of these plants, and 3) to reveal the potential health risk of Se by consumption of these plants.

\section{Experimental}

\section{Sample Collection and Preparation}

Eighty-three crop samples and 144 Chinese herb samples were collected in a mountainous area of Anhui Province, China. All the soils from the roots of the plants were collected simultaneously. The sampling region is located at the intersection of Shitai and Qimen counties (Fig. 1). It contains Dashan, Shili, Shuangkeng,

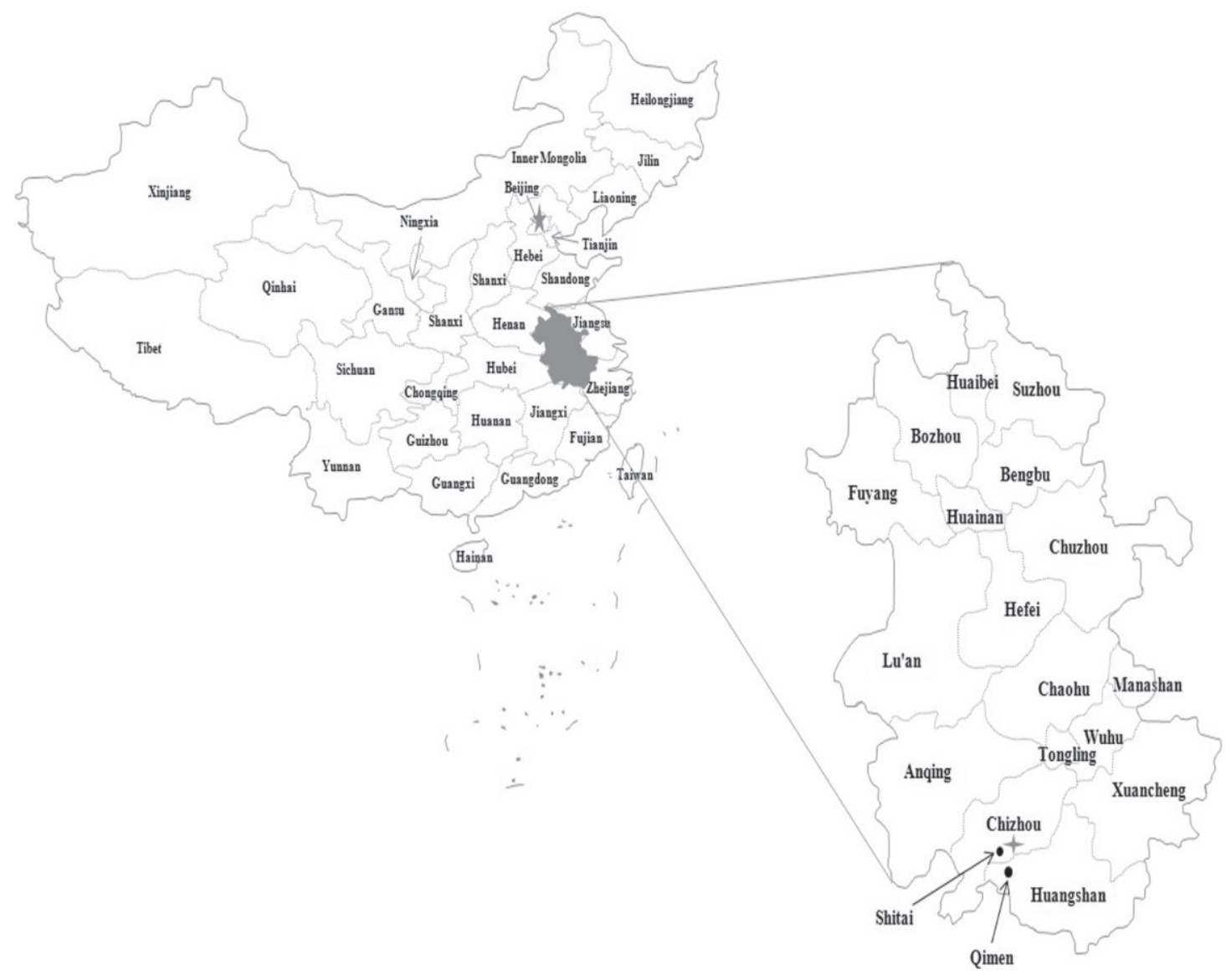

Fig. 1. Study site in Chizhou, Anhui Province. 
Table 1. Concentrations ( $\mu \mathrm{g} \mathrm{kg}^{-1}$ ) and enrichment coefficient values of Se in different crops of the Dashan Region.

\begin{tabular}{|c|c|c|c|}
\hline Crop (portion) & Concentrations of Se in crops & Concentrations of Se in soil samples & EC value \\
\hline Pumpkin (pericarp) & $120 \pm 39(\mathrm{n}=4)$ & $2000 \pm 170$ & $6.2 \% \pm 2.0 \%$ \\
\hline Fatmelon (pericarp) & $120 \pm 42(\mathrm{n}=4)$ & $860 \pm 110$ & $14 \% \pm 4.9 \%$ \\
\hline Asparagus bean (fruit) & $120 \pm 37(\mathrm{n}=4)$ & $860 \pm 170$ & $14 \% \pm 4.3 \%$ \\
\hline Luffa (fruit) & $120 \pm 31(\mathrm{n}=4)$ & $940 \pm 130$ & $13 \% \pm 3.3 \%$ \\
\hline Shallot (stem and leaf) & $170 \pm 33(\mathrm{n}=4)$ & $990 \pm 150$ & $17 \% \pm 3.3 \%$ \\
\hline Capsicum (fruit) & $500 \pm 40(\mathrm{n}=4)$ & $1400 \pm 190$ & $37 \% \pm 2.9 \%$ \\
\hline Maize (grain) & $100 \pm 53(\mathrm{n}=8)$ & $740 \pm 200$ & $14 \% \pm 7.3 \%$ \\
\hline Potato (tuber) & $110 \pm 63(\mathrm{n}=10)$ & $910 \pm 260$ & $13 \% \pm 7.5 \%$ \\
\hline Rice (grain) & $410 \pm 42(\mathrm{n}=5)$ & $1100 \pm 190$ & $39 \% \pm 3.9 \%$ \\
\hline Radish (root) & $3100 \pm 2400(\mathrm{n}=11)$ & $1200 \pm 730$ & $300 \% \pm 230 \%$ \\
\hline Eggplant (fruit) & $200 \pm 47(\mathrm{n}=4)$ & $900 \pm 200$ & $23 \% \pm 5.5 \%$ \\
\hline Fragrant-flowered garlic (stem and leaf) & $270 \pm 36(\mathrm{n}=4)$ & $890 \pm 190$ & $31 \% \pm 4.1 \%$ \\
\hline Konjac (tuber) & $230 \pm 29(\mathrm{n}=4)$ & $930 \pm 110$ & $25 \% \pm 3.2 \%$ \\
\hline Persimmon (fruit) & $210 \pm 45(\mathrm{n}=4)$ & $740 \pm 240$ & $29 \% \pm 6.3 \%$ \\
\hline Tea (leaf) & $340 \pm 58(\mathrm{n}=9)$ & $780 \pm 190$ & $45 \% \pm 7.6 \%$ \\
\hline
\end{tabular}

and Xianyu villages of Shitai County, and Liuyuan village of Qimen County(E117 $19^{\prime} 54^{\prime \prime}-117^{\circ} 24^{\prime} 53^{\prime \prime}$, $\left.\mathrm{N} 30^{\circ} 00^{\prime} 00^{\prime \prime}-30^{\circ} 03^{\prime} 06^{\prime \prime}\right)$. A portable GPS device was used to locate the sampling sites. The sampling area is about $46.22 \mathrm{~km}^{2}$. The annual average temperature of the sampling area is $16^{\circ} \mathrm{C}$. The annual average rainfall of this area is $1,626.4 \mathrm{~mm}$, and total average annual evaporation capacity is $1,256.2 \mathrm{~mm}$ [15].

In the laboratory, an edible portion of crops and a medical portion of Chinese herbs (Tables 1-2) were washed with Milli-Q water, dried in an oven at $50^{\circ} \mathrm{C}$, and then ground into powders $(0.15 \mathrm{~mm})$ in a grinder. Plant root soils were air-dried, homogeneously ground, and sieved through a $0.15 \mathrm{~mm}$ mesh. Finally, all the samples were stored at $-20^{\circ} \mathrm{C}$ before analysis.

\section{Sample Digestion and Determination}

The measurement of total Se was accomplished by the use of previously established methods [16]. Briefly, about $3 \mathrm{~g}$ of sample was digested overnight in a $50 \mathrm{~mL}$ conical flask by $10 \mathrm{~mL}$ of concentrated nitric acid and perchloric acid (4:1 v:v). The acid mixture was then heated at $100^{\circ} \mathrm{C}$ for $1 \mathrm{~h}, 120^{\circ} \mathrm{C}$ for $2 \mathrm{~h}$, and then $180^{\circ} \mathrm{C}$ for $1 \mathrm{~h}$ using an electric plate. The samples were then heated at $210^{\circ} \mathrm{C}$ until no white fume appeared. The remaining solution was cooled to room temperature, and $5 \mathrm{~mL}$ of hydrochloric acid $(\mathrm{HCl}, 12 \mathrm{M})$ was added to convert $\mathrm{Se}^{6+}$ to $\mathrm{Se}^{4+}$, for about $4 \mathrm{~h}$. Finally, the solution was adjusted to $25 \mathrm{~mL}$ with Milli-Q water for Se analysis.

All of the prepared samples were analyzed with hydride generation atomic fluorescence spectrometry (HG-AFS 9230, Beijing Titan Instrument Co., China).
HG-AFS, which uses sodium borohydride $\left(\mathrm{NaBH}_{4}\right)$ (or potassium borohydride, $\mathrm{KBH}_{4}$ ) as a reducing agent, will reduce $\mathrm{Se}^{4+}$ to hydrogen selenide $\left(\mathrm{H}_{2} \mathrm{Se}\right)$ in $\mathrm{HCl}$ solution. The irradiation of a hollow cathode lamp excites the electrons in atoms of $\mathrm{Se}$ and causes them to emit fluorescence. The strength of the fluorescence increases with the increasing content of Se. The detection limit of the HG-AFS method for samples was $0.1 \mu \mathrm{gL}^{-1}$ solution.

\section{Quality Control}

Confidence in the measurements was qualified by using blank controls, certified reference materials, and duplicates. National standard reference material GSS-1 (soil) and GSV-2 (shrub leaves) were used to check the recoveries of Se. The recoveries of the standard reference material ranged from 85 to $118 \%$, with the relative standard deviation (RSD) being 10\%. The detection limit (DL) of the instrument was $0.1 \mu \mathrm{g} \mathrm{L}^{-1}$ solution.

\section{Results and Discussion}

\section{Distribution of Se in Crops of the Dashan Region}

The concentrations and enrichment coefficients of Se in different crops of the Dashan Region can be found in Table 1. The crop with the highest levels of Se was radish, which had an average concentration of $3,100 \mu \mathrm{g} \mathrm{kg}^{-1}$, and the lowest levels of Se were found in maize samples, with the average concentration of $100 \mu \mathrm{g} \mathrm{kg}^{-1}$. The order of average concentration of Se from high to low was: radish, capsicum annuum, rice, tea, fragrant-flowered 
Table 2. Average concentrations of Se in Chinese herbs $\left(\mu \mathrm{g} \mathrm{kg}^{-1}\right)$ and enrichment coefficient values of Se in Chinese herbs of the Dashan Region.

\begin{tabular}{|c|c|c|c|c|c|c|}
\hline Family name & Latin name & $\begin{array}{l}\text { Alphabetic } \\
\text { (Ping Yin) }\end{array}$ & Medical portion & $\begin{array}{l}\text { Concentra- } \\
\text { tions of Se in } \\
\text { Chinese herbs }\end{array}$ & $\begin{array}{l}\text { Concentra- } \\
\text { tions of Se in } \\
\text { soil samples }\end{array}$ & $\begin{array}{c}\mathrm{EC} \\
\text { value }\end{array}$ \\
\hline \multirow{6}{*}{ LABIATAE } & Mosla dianthera & Xiao Yu Xian Cao & whole plant & 540 & 1200 & $44 \%$ \\
\hline & $\begin{array}{l}\text { Perilla frutescens } \\
\text { var. acuta }\end{array}$ & Bai Su & whole plant & 410 & 890 & $46 \%$ \\
\hline & Glechoma longituba & Huo Xu Dan & whole plant & 110 & 1000 & $11 \%$ \\
\hline & Perilla frutescens & $\mathrm{Zi} \mathrm{Su}$ & whole plant & 270 & 1900 & $14 \%$ \\
\hline & Leonurus artemisia & Yi Mu Cao & aboveground part & 320 & 920 & $35 \%$ \\
\hline & $\begin{array}{l}\text { Clinopodium polyc- } \\
\text { ephalum }\end{array}$ & Duan Xue Liu & aboveground part & 490 & 1000 & $48 \%$ \\
\hline \multirow{3}{*}{ ARACEAE } & Colocasia esculenta & $\mathrm{Yu}$ & tuber & 30 & 960 & $3.5 \%$ \\
\hline & Pinellia cordata & Di Shui Zhu & tuber & 720 & 1100 & $63 \%$ \\
\hline & Acorus tatarinowii & Shi Chang Pu & rhizome & 390 & 1100 & $35 \%$ \\
\hline \multirow{4}{*}{ CRASSULACEAE } & Sedum sarmentosum & Chui Pen Cao & whole plant & 360 & 910 & $39 \%$ \\
\hline & $\begin{array}{l}\text { Sedum plumbizin- } \\
\text { cicola }\end{array}$ & Ban Kuang Jing Tian & whole plant & 90 & 1100 & $8.3 \%$ \\
\hline & Sedum emarginatum & Ao Ye Jing Tian & whole plant & 50 & 1400 & $3.7 \%$ \\
\hline & Sedum shitaiense & Shi Tai Jing Tian & whole plant & 390 & 1400 & $29 \%$ \\
\hline \multirow{2}{*}{$\begin{array}{c}\text { CAPRIFOLIACE- } \\
\text { AE }\end{array}$} & Sambucus chinensis & Jie Gu Cao & whole plant & 570 & 1300 & $43 \%$ \\
\hline & Lonicera japonica & Jin Yin Hua & flower & 550 & 2000 & $27 \%$ \\
\hline \multirow{3}{*}{ ROSACEAE } & Eriobotrya japonica & $\mathrm{Pi} \mathrm{Pa}$ & fruit & 600 & 730 & $83 \%$ \\
\hline & Rubus hirsutus & Peng Lei & fruit & 70 & 1300 & $5.3 \%$ \\
\hline & Rosa laevigata & Jin Ying Zi & fruit & 210 & 1900 & $11 \%$ \\
\hline \multirow{4}{*}{ URTICACEAE } & $\begin{array}{c}\text { Elatostema involu- } \\
\text { cratum }\end{array}$ & Lou Ti Cao & whole plant & 820 & 1400 & $57 \%$ \\
\hline & Pellionia radicans & Chi Che & whole plant & 140 & 2700 & $5.3 \%$ \\
\hline & Gonostegia hirta & Nuo Mi Tuan & whole plant & 180 & 2400 & $7.7 \%$ \\
\hline & Pilea sinofasciata & $\mathrm{Cu}$ Chi Leng Shui Hua & whole plant & 310 & 680 & $46 \%$ \\
\hline \multirow{3}{*}{ COMPOSITAE } & $\begin{array}{c}\text { Dendranthema } \\
\text { indicum }\end{array}$ & Ye Ju Hua & aboveground part & 50 & 510 & $9.8 \%$ \\
\hline & Kalimeris indica & Ma Lan & whole plant & 360 & 990 & $37 \%$ \\
\hline & Gynura crepidioides & Ge Ming Cai & whole plant & 120 & 1100 & $11 \%$ \\
\hline \multirow{2}{*}{$\begin{array}{l}\text { GLEICHENIACE- } \\
\text { AE }\end{array}$} & Hicriopteris glauca & Li Bai & aboveground part & 60 & 490 & $12 \%$ \\
\hline & $\begin{array}{l}\text { Dicranopteris di- } \\
\text { chotoma }\end{array}$ & Mang Qi & aboveground part & 70 & 4400 & $1.6 \%$ \\
\hline \multirow[b]{2}{*}{ GRAMINEAE } & Coix lacrymajobi & $\mathrm{Yi} \mathrm{Yi}$ & Stem and leaf & 120 & 2400 & $4.8 \%$ \\
\hline & $\begin{array}{l}\text { Triarrhena saccha- } \\
\text { riflora }\end{array}$ & Di & rhizome & 90 & 1900 & $4.8 \%$ \\
\hline \multirow[b]{2}{*}{ UMBELLIFERAE } & Oenanthe javanica & Shui Qin & whole plant & 910 & 1600 & $56 \%$ \\
\hline & $\begin{array}{l}\text { Cryptotaenia } \\
\text { japonica }\end{array}$ & Ya Er Qin & whole plant & 190 & 1200 & $16 \%$ \\
\hline \multirow{2}{*}{$\begin{array}{c}\text { POLYGONACE- } \\
\text { AE }\end{array}$} & Reynoutria japonica & Hu Zhang & aboveground part & 290 & 1900 & $15 \%$ \\
\hline & Rumex japonicus & Yang Ti & whole plant & 1500 & 2600 & $56 \%$ \\
\hline
\end{tabular}


Table 2. Continued.

\begin{tabular}{|c|c|c|c|c|c|c|}
\hline \multirow{2}{*}{ LILIACEAE } & Smilax glabra & Tu Fu Ling & rhizome & 270 & 1200 & $23 \%$ \\
\hline & Tricyrtis macropoda & You Dian Cao & root & 130 & 1200 & $11 \%$ \\
\hline MORACEAE & Ficus pumila & $\mathrm{Pi} \mathrm{Li}$ & stem and leaf & 470 & 1300 & $36 \%$ \\
\hline $\begin{array}{l}\text { VALERIANACE- } \\
\text { AE }\end{array}$ & Patrinia villosa & Bai Hua Bai Jiang & whole plant & 230 & 980 & $24 \%$ \\
\hline ARALIACEAE & Aralia chinensis & Cong $\mathrm{Mu}$ & stem & 250 & 2700 & $9.3 \%$ \\
\hline ACTINIDIACEAE & Actinidia chinensis & $\begin{array}{c}\text { Zhong Hua Mi Hou Tao } \\
\text { Ye }\end{array}$ & leaf & 600 & 2500 & $25 \%$ \\
\hline SAURURACEAE & Houttuynia cordata & Yu Xing Cao & whole plant & 280 & 2100 & $13 \%$ \\
\hline OSMUNDACEAE & Osmunda japonica & Zi Qi & aboveground part & 1000 & 2300 & $44 \%$ \\
\hline THEACEAE & Camellia oleifera & You Cha & leaf & 780 & 2400 & $32 \%$ \\
\hline $\begin{array}{c}\text { EUPHORBIACE- } \\
\text { AE }\end{array}$ & Sapium sebiferum & Wu Jiu & branch and leaf & 800 & 680 & $117 \%$ \\
\hline ERICACEAE & Lyonia ovalifolia & Mi Fan Hua & branch and leaf & 160 & 830 & $19 \%$ \\
\hline LAURACEAE & Lindera aggregata & Wu Yao & branch and leaf & 110 & 1020 & $10 \%$ \\
\hline $\begin{array}{l}\text { PLANTAGI- } \\
\text { NACEAE }\end{array}$ & Plantago asiatica & Che Qian & whole plant & 520 & 1140 & $46 \%$ \\
\hline JUNCACEAE & Juncus effusus & Deng Xing Cao & whole plant & 510 & 960 & $53 \%$ \\
\hline ACANTHACEAE & $\begin{array}{l}\text { Rostellularia } \\
\text { procumbens }\end{array}$ & Jue Chuang & whole plant & 300 & 1400 & $21 \%$ \\
\hline $\begin{array}{l}\text { CHLORAN- } \\
\text { THACEAE }\end{array}$ & Chloranthus spicatus & Jin Li Lan & whole plant & 170 & 1300 & $14 \%$ \\
\hline MYRSINACEAE & Ardisia crenata & Zhu Sha Gen & whole plant & 220 & 1100 & $20 \%$ \\
\hline GESNERIACEAE & $\begin{array}{l}\text { Lysionotus pauci- } \\
\text { florus }\end{array}$ & Diao Shi Ju Tai & branch & 470 & 980 & $48 \%$ \\
\hline $\begin{array}{l}\text { AMARYLLI- } \\
\text { DACEAE }\end{array}$ & Lycoris radiata & Shi Suan & bulb & 190 & 1000 & $19 \%$ \\
\hline $\begin{array}{l}\text { AQUIFOLIACE- } \\
\text { AE }\end{array}$ & Ilex latifolia & Ku Ding Cha & Leaf & 100 & 850 & $12 \%$ \\
\hline VERBENACEAE & Premna microphylla & Dou Fu Chai & branch and leaf & 110 & 2100 & $4.9 \%$ \\
\hline $\begin{array}{l}\text { DIOSCOREACE- } \\
\text { AE }\end{array}$ & Dioscorea bulbifer & Huang Du & tuber & 660 & 1800 & $38 \%$ \\
\hline MYRSINACEAE & Ardisia crispa & Bai Liang Jin & whole plant & 40 & 470 & $8.5 \%$ \\
\hline HUPERZIACEAE & Huperzia serrata & She Zu Shi Shan & whole plant & 360 & 1500 & $24 \%$ \\
\hline DRYNARIACEAE & Drynaria roosii & Hu Jue & whole plant & 100 & 1400 & $7.2 \%$ \\
\hline $\begin{array}{l}\text { SAXIFRAGACE- } \\
\text { AE }\end{array}$ & Saxifraga stolonifera & $\mathrm{Hu} \mathrm{Er} \mathrm{Cao}$ & whole plant & 370 & 1400 & $26 \%$ \\
\hline $\begin{array}{l}\text { CUCURBITA- } \\
\text { CEAE }\end{array}$ & $\begin{array}{l}\text { Gynostemma penta- } \\
\text { phyllum }\end{array}$ & Jiao Gu Lan & stem and leaf & 670 & 1000 & $66 \%$ \\
\hline $\begin{array}{l}\text { LYCOPODIACE- } \\
\text { AE }\end{array}$ & $\begin{array}{l}\text { Lycopodium japoni- } \\
\text { cum }\end{array}$ & Shi Song & stem and leaf & 820 & 2300 & $36 \%$ \\
\hline CRUCIFERAE & Cardamine hirsuta & Sui Mi Ji & whole plant & 800 & 1400 & $56 \%$ \\
\hline $\begin{array}{l}\text { BERBERIDACE- } \\
\text { AE }\end{array}$ & $\begin{array}{l}\text { Epimedium sagit- } \\
\text { tatum }\end{array}$ & San Zhi Jiu Ye Cao & whole plant & 60 & 850 & $7.1 \%$ \\
\hline $\begin{array}{l}\text { SYMPLOCACE- } \\
\text { AE }\end{array}$ & Symplocos stellaris & Lao Shu Shi & branch & 410 & 960 & $43 \%$ \\
\hline PRIMULACEAE & Lysimachia fortunei & Xing Xiu Cai & whole plant & 390 & 2100 & $18 \%$ \\
\hline
\end{tabular}


Table 2. Continued.

\begin{tabular}{|c|c|c|c|c|c|c|}
\hline Family name & Latin name & $\begin{array}{c}\text { Alphabetic } \\
\text { (Ping Yin) }\end{array}$ & Medical portion & $\begin{array}{c}\text { Concentra- } \\
\text { tions of Se in } \\
\text { Chinese herbs }\end{array}$ & $\begin{array}{c}\text { Concentra- } \\
\text { tions of Se in } \\
\text { soil samples }\end{array}$ & $\begin{array}{c}\text { EC } \\
\text { value }\end{array}$ \\
\hline $\begin{array}{c}\text { SELAGINEL- } \\
\text { LACEAE }\end{array}$ & $\begin{array}{c}\text { Selaginella moellen- } \\
\text { dorffi }\end{array}$ & Jiang Nan Chun Bai & whole plant & 130 & 450 & $29 \%$ \\
\hline $\begin{array}{c}\text { POLYPODIACE- } \\
\text { AE }\end{array}$ & $\begin{array}{c}\text { Polypodium nip- } \\
\text { ponicum }\end{array}$ & Shui Long Gu & whole plant & 160 & 880 & $18 \%$ \\
\hline SOLANACEAE & Nicotiana tabacum & Yan Ye & whole plant & 770 & 1000 & $75 \%$ \\
\hline RUBIACEAE & $\begin{array}{c}\text { Gardenia jasmi- } \\
\text { noides }\end{array}$ & Zhi Zi & branch & 20 & 840 & $2.2 \%$ \\
\hline THUIDIACEAE & $\begin{array}{c}\text { Thuidium cymbifo- } \\
\text { lium }\end{array}$ & Da Yu Xian & whole plant & 1100 & 1500 & $71 \%$ \\
\hline CYPERACEAE & Cyperus rotundus & Suo Cao & whole plant & 720 & 790 & $91 \%$ \\
\hline PTERIDACEAE & $\begin{array}{c}\text { Pteridium aquilinum } \\
\text { var.latiusculum }\end{array}$ & \multicolumn{2}{|c|}{ Jue plant } & 260 & 2400 & $11 \%$ \\
\hline
\end{tabular}

garlic, konjac, persimmon, shallot, pumpkin, fatmelon, asparagus bean, luffa, potato, and maize.

In the Dashan Region, paddy rice is the primary cereal, and it accounts for a significant proportion of daily dose of protein and micronutrients. Distribution of $\mathrm{Se}$ in rice from China and all over the world can be found in Fig. 2 [2]. In China, the mean value of Se in rice is about $88 \mu \mathrm{g} \mathrm{kg}^{-1}$. However, the concentrations found in the rice samples of the Dashan Region $\left(410 \pm 42 \mu \mathrm{g} \mathrm{kg}^{-1}\right)$ were much higher than this value, and was also higher than the mean global value $\left(95 \mu \mathrm{g} \mathrm{kg}^{-1}\right)$. Compared with previous measurements of Se in rice samples of other regions of China, the levels of $\mathrm{Se}$ in rice samples of the Dashan Region were relatively higher than those found in Kaiyang of Guizhou Province (mean $=230 \mu \mathrm{g} \mathrm{kg}^{-1}$ ) [17], the Three Gorges Reservoir Region of Chongqing $\left(\right.$ mean $\left.=55 \mu \mathrm{g} \mathrm{kg}^{-1}\right)$ [18], Hanyin of Shanxi Province (mean $=172 \mu \mathrm{g} \mathrm{kg}^{-1}$ ) [19], and Suzhou of Jiangsu Province $\left(\right.$ mean $\left.=23.6 \mu \mathrm{g} \mathrm{kg}^{-1}\right)$ [16], but was about 2 times lower than that found in rice samples of Enshi of Hubei Province $\left(\right.$ mean $=960 \mu \mathrm{g} \mathrm{kg}^{-1}$ ), which was recognized as the "world capital of Se" [20]. Furthermore, Se in other crops of the Dashan region, such as maize, potato, pumpkin, eggplant, capsicum, and radish, were also obviously higher than those found in crops of other regions of China, but were lower than those detected in crops of Enshi. The details can be found in Table 3 [7, 16-21]. Generally, levels of Se in crops of the Dashan region were at the high end of China. According to the classification of Tan [22], crops in China could be classified into 5 different groups by the levels of Se: deficient $\left(<25 \mu \mathrm{g} \mathrm{kg}^{-1}\right)$, marginal $\left(25-40 \mu \mathrm{g} \mathrm{kg}^{-1}\right)$, moderately high (40-70 $\left.\mu \mathrm{g} \mathrm{kg}^{-1}\right)$, high $\left(70-1,000 \mu \mathrm{g} \mathrm{kg}^{-1}\right)$, and excessive $\left(1,000+\mu \mathrm{g} \mathrm{kg}^{-1}\right)$. According to the classification, about $92 \%$ of crops in the Dashan Region presented high levels of Se, such as rice, tea, eggplant, and capsicum, etc., and the other $7.7 \%$ of crops had excessive levels of Se, such as radish (Fig. 3).

\section{Distribution of Se in Chinese Herbs of the Dashan Region}

Chinese herbs have been used for centuries in China, and more than 300 herbs are commonly used today. Dashan is one of the most important Chinese herb production areas of Anhui Province. In order to reveal the levels of Se in Chinese herbs of Dashan, 144 Chinese herb samples were collected (72 species with duplication).

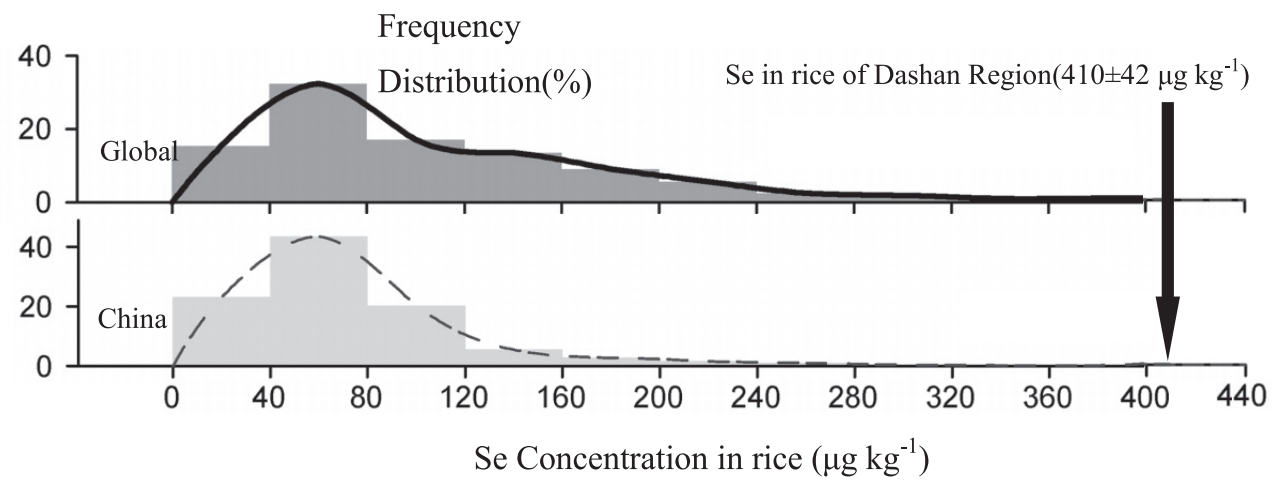

Fig. 2. Se Distribution in rice from China and globally [2]. 
Table 3. Comparison with a previous study of concentrations $\left(\mu \mathrm{g} \mathrm{kg}^{-1}\right)$ and enrichment coefficient (EC value, \%) values of Se in the crops and Chinese herbs of the Dashan Region.

\begin{tabular}{|c|c|c|c|c|c|}
\hline Research area & Types & Concentrations of Se & EC value & Sampling date & Reference \\
\hline \multicolumn{6}{|c|}{ Crops and Vegetables } \\
\hline Dashan, Anhui, China & Rice $(\mathrm{n}=5)$ & $410(365-480)$ & $39(34-45)$ & 2013 & This study \\
\hline Suzhou,Jiangsu, China & $\operatorname{Rice}(\mathrm{n}=21)$ & $23.6(15 \sim 35)$ & - & 2011 & {$[16]$} \\
\hline Kaiyang, Guizhou, China & $\operatorname{Rice}(\mathrm{n}=5)$ & $230(74 \sim 710)$ & - & 2013 & [17] \\
\hline $\begin{array}{c}\text { Three Gorges Reservoir Region, } \\
\text { Chongqing, China }\end{array}$ & $\operatorname{Rice}(\mathrm{n}=225)$ & $55(2 \sim 170)$ & - & 2006 & {$[18]$} \\
\hline Hanyin, Shanxi, China & $\operatorname{Rice}(n=4)$ & 172(137 202) & $(0.4 \sim 30)$ & 2013 & [19] \\
\hline Enshi, Hubei, China & $\operatorname{Rice}(\mathrm{n}=66)$ & $960(-)$ & - & 2013 & [20] \\
\hline Dashan, Anhui, China & Maize $(\mathrm{n}=8)$ & $100(35-200)$ & $14(7.1-20)$ & 2013 & This study \\
\hline Ping'an,Qinghai, China & Maize $(n=4)$ & $70(24 \sim 87)$ & - & 2012 & [7] \\
\hline Kaiyang, Guizhou, China & Maize $(n=5)$ & $190(53 \sim 360)$ & - & 2013 & {$[17]$} \\
\hline $\begin{array}{c}\text { Three Gorges Reservoir Region, } \\
\text { Chongqing, China }\end{array}$ & $\operatorname{Maize}(\mathrm{n}=225)$ & $42(2 \sim 103)$ & - & 2006 & {$[18]$} \\
\hline Hanyin, Shanxi, China & $\operatorname{Maize}(n=4)$ & $14(6 \sim 31)$ & - & 2013 & [19] \\
\hline Enshi, Hubei, China & $\operatorname{Maize}(\mathrm{n}=124)$ & $430(-)$ & - & 2013 & [20] \\
\hline Dashan, Anhui, China & Potato $(n=10)$ & $110(50-212)$ & $13(6.5-28)$ & 2013 & This study \\
\hline Ping'an, Qinghai, China & Potato $(\mathrm{n}=31)$ & $8.4(3 \sim 22)$ & - & 2012 & [7] \\
\hline Suzhou,Jiangsu, China & Potato $(n=21)$ & $6(4 \sim 11)$ & - & 2011 & [16] \\
\hline Enshi, Hubei, China & Potato $(\mathrm{n}=7)$ & $280(40 \sim 1070)$ & - & 2013 & {$[20]$} \\
\hline Dashan, Anhui, China & Pumpkin $(\mathrm{n}=4)$ & $120(69-165)$ & $6.2(3.8-8.6)$ & 2013 & This study \\
\hline Ping'an,Qinghai, China & Pumpkin(n=5) & $17(6 \sim 21)$ & - & 2012 & [7] \\
\hline Hanyin, Shanxi, China & Pumpkin(n=2) & $113(72 \sim 155)$ & - & 2013 & [19] \\
\hline Enshi, Hubei, China & Pumpkin(n=9) & $760(310 \sim 3200)$ & - & 2013 & [20] \\
\hline Dashan, Anhui, China & Eggplant $(n=4)$ & $200(140-257)$ & $23(16-29)$ & 2013 & This study \\
\hline Suzhou,Jiangsu, China & $\operatorname{Eggplan}(\mathrm{n}=21)$ & $1.7(0.8 \sim 3.9)$ & - & 2011 & {$[16]$} \\
\hline Kaiyang, Guizhou, China & Eggplant(n=3) & $46(23 \sim 78)$ & - & 2013 & {$[17]$} \\
\hline Enshi, Hubei, China & Eggplant(n=9) & 1040(430 3200) & - & 2013 & {$[20]$} \\
\hline Leping, Jiangxi, China & Eggplant(n=3) & $17.5(9 \sim 27)$ & 0.029 & 2012 & [21] \\
\hline Dashan, Anhui, China & Capsicum $(\mathrm{n}=4)$ & $500(443-540)$ & $37(33-40)$ & 2013 & This study \\
\hline Kaiyang, Guizhou, China & Capsicum(n=6) & $150(67 \sim 330)$ & - & 2013 & {$[17]$} \\
\hline Leping, Jiangxi, China & Capsicum(n=3) & $17(9 \sim 25)$ & 0.037 & 2012 & {$[21]$} \\
\hline Dashan, Anhui, China & $\begin{array}{l}\text { Radish (root) } \\
\quad(\mathrm{n}=11)\end{array}$ & $3100(680-8262)$ & $\begin{array}{c}300(133- \\
901)\end{array}$ & 2013 & This study \\
\hline Kaiyang, Guizhou, China & $\operatorname{Radish}(\mathrm{n}=3)$ & $180(78 \sim 260)$ & - & 2013 & {$[17]$} \\
\hline \multicolumn{6}{|c|}{ Chinese herbs } \\
\hline Dashan, Anhui, China & $-(n=144)$ & $370(20 \sim 1500)$ & $29(1.6-117)$ & & This study \\
\hline $\begin{array}{l}\text { Minjiang Estuary Wetland, Fujian, } \\
\text { China }\end{array}$ & $-(-)$ & $-(17 \sim 85)$ & $27(16 \sim 35)$ & - & {$[23]$} \\
\hline Xishuangbanna, Yunnan, China & $-(-)$ & $85(-)$ & - & - & [24] \\
\hline Salek valley, Slovenia & $-(-)$ & $140(10 \sim 320)$ & - & - & {$[25]$} \\
\hline Baoding, Hebei, China & $-(n=7)$ & $90(43 \sim 210)$ & - & - & {$[26]$} \\
\hline Liaoning, China & $-(-)$ & $140(90 \sim 210)$ & - & - & [27] \\
\hline
\end{tabular}

† average (range) 


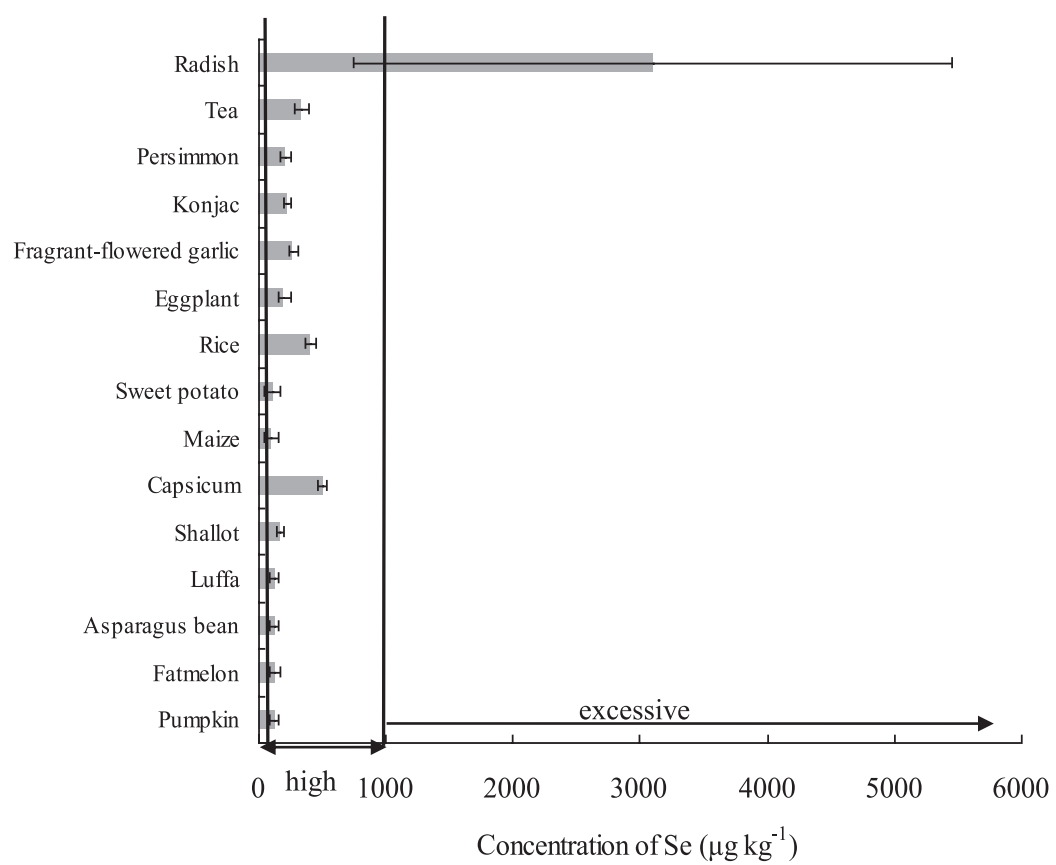

Fig. 3. Levels of Se in different crops of the Dashan Region.

The highest levels of Se were found in rumex japonicus, with an average concentration of $1,500 \mu \mathrm{g} \mathrm{kg}^{-1}$, while the lowest concentrations of Se were detected in cape jasmine, with an average concentration of $20 \mu \mathrm{g} \mathrm{kg}^{-1}$. The average concentration of $\mathrm{Se}$ in all Dashan herbs of was $370 \mu \mathrm{g} \mathrm{kg}^{-1}$.

Compared with previous studies carried out in China, the levels of Se in Dashan herbs were higher than those found in Xishuangbanna of Yunnan Province (average concentration of $85 \mu \mathrm{g} \mathrm{kg}^{-1}$ ), Liaoning Province (average concentration of $143 \mu \mathrm{g} \mathrm{kg}^{-1}$ ), Baoding of Hebei Province (average concentration of $90 \mu \mathrm{g} \mathrm{kg}^{-1}$ ), and the forest region of Heilongjiang and Jilin (average concentration of $196 \mu \mathrm{g} \mathrm{kg}^{-1}$ ). Furthermore, Se in Dashan herbs were also higher than those found in Slovenia (average concentration of $140 \mu \mathrm{g} \mathrm{kg}^{-1}$ ) [23-27]. In general, Dashan herbs were found to be much more enriched with Se than those in other regions of China.

\section{Accumulation of Se in Dashan Plants}

In order to reveal the ability to accumulate Se of diverse plants, enrichment coefficients (ECs) of Se were calculated with the equation $\mathrm{EC}=\mathrm{C}_{\text {Se-plant }} / \mathrm{C}_{\text {Se-soil }}$ [28], where $\mathrm{C}_{\text {Se-plant }}$ is the concentration of total Se in the plant $\left(\mu \mathrm{g} \mathrm{kg}{ }^{-1}, \mathrm{DW}\right)$, and $\mathrm{C}_{\mathrm{Se}-\text { soil }}$ is the concentration of total $\mathrm{Se}$ in the soil ( $\left.\mu \mathrm{g} \mathrm{kg}^{-1}, \mathrm{DW}\right)$.

The details of ECs of Se in crops and Dashan herbs can be found in Tables 1-2. The mean values of ECs of Se in diverse crops were in the range $6.1-300 \%$ with an arithmetic mean of $41 \%$, while the mean values in Chinese herbs were in the range $1.6-117 \%$ with an arithmetic mean of $29 \%$. Generally, the ECs of Se in crops were relatively higher than those found in Chinese herbs. For different kinds of crops, relatively high ECs of Se were found in radish, tea, and rice, with mean values of $300 \%, 45 \%$, and $38 \%$, respectively. For Chinese herbs, highest ECs of Se were found in Sapium sebiferum (117\%).

Compared with previous studies of China, the ECs of Se in Dashan rice (ranging from $34 \%$ to $45 \%$ ) were much higher than those found in Hanying of Shanxi Province, which ranged from $0.4 \%$ to $30 \%$ [19]. The ECs of Se in Dashan eggplant (16\% to $29 \%$ ) and capsicum (33\% to $40 \%$ ) were higher than those found in Leping of Jiangxi Province, in which the mean EC values of Se in eggplant and capsicum were 0.029 and 0.037 , respectively (Table 3) [21]. For Chinese herbs, the ECs of Se found in the Dashan Region were also higher than those found in Minjiang Estuary Wetland of Fujian Province [23]. Overall, the crops and Chinese herbs not only had the relatively high levels of Se but also had the better ability to accumulate Se from soil.

\section{Daily Intake of Se from Plants of the Dashan Region}

Food is the major resource of Se intake for humans. Dietary intake varies with the geographical source of the foods and the eating habits of the residents. In contrast to many other micronutrients, the intake of Se varies hugely worldwide, ranging from deficient (associated with selenium-deficiency diseases) to toxic concentrations that cause garlic breath, hair and nail loss, disorders of the nervous system and skin, poor dental health, and paralysis [4]. The recommended dietary allowance (RDA) of Se for humans varies by country, region, age, and sex. The RDA value for Se is $55 \mu \mathrm{g} \mathrm{day}^{-1}$ in the United States, Canada, and Europe. In China, the RDA value is $60 \mu \mathrm{g}$ day $^{-1}$. In Australia and New Zealand, the RDA values for adult men and women are 60 and $55 \mu$ day $^{-1}$, respectively. 
Table 4. Comparison with a previous study of estimated selenium intake of adults in the Dashan Region.

\begin{tabular}{|c|c|c|}
\hline Country & Se intake $(\mu \mathrm{g} /$ person per d) & Reference \\
\hline \multicolumn{3}{|c|}{ China } \\
\hline Dashan Region & $280 \pm 20$ & This study \\
\hline Belgium & 60 & {$[30]$} \\
\hline Switzerland & 66 & {$[31]$} \\
\hline Japan & 82.7 (mountain community & {$[32]$} \\
\hline Slovenia & 118 ( coastal community) & {$[33]$} \\
\hline Korea & 87 & {$[34]$} \\
\hline Greece & 58 & {$[35]$} \\
\hline Saudi Arabia & $75 \sim 122$ & {$[36]$} \\
\hline
\end{tabular}

In the UK, the reference nutrient intake is set at 75 and $60 \mu \mathrm{g} \mathrm{day}^{-1}$ for adult men and women, respectively. The RDA values suggested by WHO for adult men and women are 34 and $26 \mu \mathrm{g} \mathrm{day}^{-1}$ [4]. The tolerable upper Se intake level for adults is $400 \mu \mathrm{g}$ day $^{-1}$ [4].

Concentrations of Se in the crops were used to conduct human health risk assessments. The estimated daily intake (EDI, $\mu \mathrm{g} \mathrm{day}^{-1}$ ) of Se for the general population was calculated using the following equation: $\mathrm{EDI}=\mathrm{C} \times \mathrm{C}_{\mathrm{R}}$, where $\mathrm{C}$ is the mean concentration of $\mathrm{Se}$ in each species $\left(\mu \mathrm{g} \mathrm{kg}^{-1}\right)$ and $\mathrm{C}_{\mathrm{R}}$ represents the consumption of crops per capita per day $\left(\mathrm{kg} \mathrm{day} \mathrm{y}^{-1}\right)$. The daily diet of residents in the Dashan Region from plants mainly contains $0.5 \mathrm{~kg}$ of cereals and $0.378 \mathrm{~kg}$ of vegetables [29]. According to the composition of residents' daily diet and the Se concentrations of different crops in the Dashan Region, the estimated daily Se intake from plants per capita was $280 \pm 20 \mu \mathrm{g} \mathrm{day}^{-1}$. In this case, the content of Se in crops could satisfy daily Se intake sufficiently, while the daily Se dietary intake also is lower than the tolerable upper Se intake level [4]. Compared with other regions of China, the EDI of Se for Dashan residents was higher than those found in the Keshan disease area and the moderate $\mathrm{Se}$ area, but were lower than those in the selenosis area. Compared with other countries, the EDI of $\mathrm{Se}$ in the Dashan was higher than those in Belgium, Switzerland, Japan, Korea, Slovenia, Greece, and Saudi Arabia [30-36]. In summary, the EDI of Se in Dashan was at the highest level of the world (Table 4). Based on this research, Serich agricultural products could be developed in the Dashan Region and the crops that had strong enrichment ability of Se could be utilized in low-Se areas to produce Se-rich foodstuffs and thereby increase health benefits in the Se deficiency areas.

\section{Conclusions}

The levels of selenium ranged from 100 to $3,100 \mu \mathrm{g} \mathrm{kg}^{-1}$ (DW) in crops, and 20 to $1,500 \mu \mathrm{g} \mathrm{kg}^{-1}$ in
Chinese herbs. The Enrichment coefficient values were $6.1-300 \%$ in crops and $1.6-117 \%$ in Chinese herbs of the Dashan Region. The crops and Chinese herbs not only had relatively high levels of Se but also had the better ability to accumulate Se from soil. The estimated daily Se intake per capita from crops was $282 \pm 20 \mu \mathrm{g} \mathrm{day}^{-1}$. The content of Se in crops could satisfy daily Se intake sufficiently, while the daily Se dietary intake also was lower than the tolerable upper Se intake level.

\section{Acknowledgements}

This study was supported by grants from the National Natural Science Foundation of China (project No. 41203075), the Natural Science Foundation of Anhui Province (No. 1308085QB31), the Science and Technology Research Foundation of Anhui Province (No. 12010402133), the Research Culture Funds of Anhui Normal University (No. 2013rcpy47), and the Innovation and Entrepreneurship Training Program of Anhui Normal University (No. 201610370462).

\section{References}

1. COPPINGER R.J., DIAMOND A.M. Selenium: its molecular biology and role in human health., Kluwer Academic Press; 219, Netherlands, 2001.

2. WILLIAMS P.N., LOMBI E., SUN G.X., SCHECKEL K., ZHU Y.G., FENG X.B., ZHU J.M., CAREY A.M., ADOMAKO E., LAWGALI Y., DEACON C., MEHARG A.A. Selenium characterization in the global rice supply chain. Environmental Science \& Technology. 43 (15), 6024, 2009.

3. RAYMAN M.P. The importance of selenium to human health. Lancet. 356 (9225), 233, 2000.

4. RAYMAN M.P. Selenium and human health. Lancet. 379 (9822), 1256, 2012.

5. LI T.Y., YUAN X.Y., SONG Y.X., CHEN H.Y., LIU Q., HU S. Infuence of Heavy Metals and Nutrient Concentrations on Selenium Geochemical Behavior in SoilRice System. Polish Journal of Environmental Studies. 25 (1), 185, 2016.

6. MISTRY H.D., PIPKIN F.B., REDMAN C.W.G., POSTON L. Selenium in reproductive health. American Journal of Obstetrics and Gynecology. 206 (1), 21, 2012.

7. YU D.S., LIANG D.L., LEI L.M., ZHANG R., SUN X.F., LIN Z.Q. Selenium geochemical distribution in the environment and predicted human daily dietary intake in northeastern Qinghai, China. Environmental Science \& Pollution Research. 22 (15), 11224, 2015.

8. NI R.X., LUO K.L., TIAN X.L., YAN S.G., ZHONG J.T., LIU M.Q. Distribution and geological sources of selenium inenvironmental materials in Taoyuan County,Hunan Province, China. Environmental Geochemistry \& Health. 38 (3), 927, 2016.

9. MOLNAR J. Selenium: its antioxidant effects and issues in selenium supply. Orvosi Hetilap. 154 (41), 1613, 2013.

10. KLAPCINSKA B., POPRZECKI S., DANCH A. Blood Selenium Concentration of Residents of Upper Silesia: Relation to Age and Gender. Polish Journal of Environmental Studies. 15 (5), 753, 2006 
11. ABDELHAFEZ A.A., ABBAS M.H.H., ATTIA T.M.S. Environmental Monitoring of Heavy-Metals Status and Human Health Risk Assessment in the Soil of Sahl ElHessania Area, Egypt. Polish Journal of Environmental Studies. 24 (2), 459, 2015.

12. KESKINEN R., TURAKAINEN M., HARTIKAINEN H. Plant availability of soil selenate additions and selenium distribution within wheat and ryegrass. Plant \& Soil. 333 (1-2), 301, 2010.

13. PYRZYNSKA K. Selenium speciation in enriched vegetables. Food Chemistry. 114 (4), 1183, 2009.

14. WU Y.D., XIANG F., MA L., CHEN B., SHI Q.H. The geochemistry study of selenium in the stonemountain area of Anhui Province (in Chinese). Miner Petrol. 27(4), 53, 2007.

15. XIN K., ZHOU S.B., WU X.G., ZHU Y.Y., KONG J.J., SHAO T., TAO X. Concentrations and characteristics of selenium in soil samples from Dashan Region, a seleniumenriched area in China. Soil Science \& Plant Nutrition. 61 (6), 889, 2015.

16. GAO J., LIU Y., HUANG Y., LIN Z.Q., BANUELOS G.S., LAM M.H.W., YIN X.B. Daily selenium intake in a moderate selenium deficiency area of Suzhou, China. Food Chemistry. 126 (3), 1088, 2011

17. TONG S., LIAN G., DAI W. Analysis on the selenium contents level and restriction factors in the crops in Kaiyang. Guangdong Agricultural Sciences. 35 (1), 27, 2013 [In Chinese].

18. LI Q.Y., WANG J., LI Q.L., LI X.M., HE Y.J. Analysis of the contents of 5 trace elements in the soil foodstuff and drinking water in the Three Gorges Reservoir Region. Journal of Southwest Agricultural University. 28(5), 727, 2006 [In Chinese]..

19. YU Z. Studies on main crops capacity of enrichment selenium and selenium distribution, existing form in agricultural products in Se-enriched regions of Hanyin Shanxi, Shanxi normal university; 3, China, 2013 [In Chinese].

20. HUANG Y., WANG Q.X., GAO J., LIN Z.Q., BANUELOS G.S., YUAN L.X., YIN X.B. Daily Dietary Selenium Intake in a High Selenium Area of Enshi, China. Nutrients. 5 (3), 700, 2013.

21. WU D., FANG K.M., SHEN H.F., WAN T., LU J. Comparative study on selenium-enriching ability of different types of vegetable in Leping city of Jiangxi. Acta Agriculturae Jiangxi. 24 (7), 23, 2012 [In Chinese]..

22. TAN J.A. Chronic Keshan disease and environmental elements of life (in Chinese)., Chinese Medicine Science and Technology Press; 95, China, 1996.

23. SONG P., FENG L., HONG W., WU C.Z., WANG G., LI J., HUANG L. Study on content and distribution of selenium in the soil-plant systems of Minjiang Estuary Wetland. Journal of Huaqiao University. 30 (6), 673, 2009 [In Chinese].

24. XU Y.K., LIU H.M., XIAO C.F., CHAN Y., WU Z.L., DAO X.S., CAI C.T. An analysis of selenium contents of wild vegetable in Xishuangbanna. Acta Botanica Yunnanica. 26 (6), 668, 2004 [In Chinese].

25. SLEKOVEC M., GOESSLER W. Accumulation of selenium in natural plants and selenium supplemented vegetable and selenium speciation by HPLC-ICPMS. Chemical Speciation \& Bioavailability. 17 (2), 63, 2005.

26. LIU Z.F., SUN H.W., SHEN S.G., LI L.Q., SHI H.M. Simultaneous determination of total arsenic and total selenium in Chinese medicinal herbs by hydride generation atomic fluorescence spectrometry in tartaric acid medium. Analytical Chimica Acta. 550 (1-2), 151, 2005.

27. GAO T.S., SHI R., QI T.C., YIN H.S., MEI L., HAN X.Q., CUI P. A comparative study on the effects of excess Iodine and herbs with excess Iodine on thyroid oxidative stress in Iodine-deficient rats. Biological Trace Element Research. 157 (2), 130, 2014.

28. BITTERLI C., BANUELOS G.S., SCHULIN R. Use of transfer factors to characterize uptake of selenium by plants. Journal of Geochemical Exploration. 107 (2), 206, 2010.

29. YUAN B.J., PAN X.Q., DAI Y., SHI Z.M. Investigate the change of diet and nutrition of residents in Jiangsu Province. Acta Nutrimenta Sinica. 29 (6), 569, 2007 [In Chinese]..

30. WAEGENEERS N., THIRY C., DE TEMMERMAN L., RUTTENS A. Predicted dietary intake of selenium by the general adult population in Belgium. Food Additives \& Contaminants. Part A, Chemistry, Analysis, Control, Exposure \& Risk Assessment. 30 (2), 278, 2013.

31. JENNY-BURRI J., HALDIMANN M., DUDLER V. Estimation of selenium intake in Switzerland in relation to selected food groups. Food Additives \& Contaminants. Part A, Chemistry, Analysis, Control, Exposure \& Risk Assessment. 27 (11), 1516, 2010.

32. MIYAZAKI, Y., KOYAMA H., SASADA Y., SATOH H., NOJIRI M., SUZUKI S. Dietary habits and selenium intake of residents in mountain and coastal communities in Japan. Journal of Nutritional Science and Vitaminology. 50 (50), 309, 2004

33. SMRKOLJ P., POGRAJC L., HLASTAN-RIBIC C., STIBILJ, V. Selenium content in selected Slovenian foodstuffs and estimated daily intakes of selenium. Food Chemistry. 90 (4), 691, 2005.

34. CHOI Y.,KIM J., LEE H.S., KIM C.I., HWANG I.K., PARK H.K., OH C.H . Selenium content in representative Korean foods. Journal of Food Composition \& Analysis. 22 (2), 117, 2009.

35. PAPPA E.C., PAPPAS A.C., SURAI P.F. Selenium content in selected foods from the Greek market and estimation of the daily intake. Science of the Total Environment. 372 (1), 100, 2006.

36. AL-AHMARY, KM. Selenium content in selected foods from the Saudi Arabia market and estimation of the daily intake. Arabian Journal of Chemistry. 2 (2), 95, 2009. 